\title{
A prospective evaluation of whole-exome sequencing as a first-tier molecular test in infants with suspected monogenic disorders
}

\author{
Zornitza Stark, MD1, Tiong Y. Tan, MD, PhD ${ }^{1,2}$, Belinda Chong, PhD1, Gemma R. Brett, MSc, \\ MGenCouns ${ }^{1,5}$, Patrick Yap, MD11, Maie Walsh, MD1 Alison Yeung, MD1', Heidi Peters, MD, PhD 1,2,4, \\ Dylan Mordaunt, MD ${ }^{1,2,4}$, Shannon Cowie, BSc ${ }^{1}$, David J. Amor, MD, PhD ${ }^{1,2}$, Ravi Savarirayan, MD ${ }^{1,2}$, \\ George McGillivray, MD1, Lilian Downie, MD1, Paul G. Ekert, MD, PhD 1,2, Christiane Theda, MD, PhD ${ }^{1-3}$, \\ Paul A. James, MD, PhD ${ }^{6}$, Joy Yaplito-Lee, $\mathrm{MD}^{1,4}$, Monique M. Ryan, MD ${ }^{1,2,4}$, \\ Richard J. Leventer, MD, PhD ${ }^{1,2,4}$, Emma Creed, MGenCouns ${ }^{1,5,6}$, Ivan Macciocca, BSc, MHSc ${ }^{1}$, \\ Katrina M. Bell, PhD ${ }^{1}$, Alicia Oshlack, PhD ${ }^{1,2}$, Simon Sadedin, BSc, BEng ${ }^{1}$, \\ Peter Georgeson, BEng, BMath², Charlotte Anderson, BSc, MRes², Natalie Thorne, PhD 1,2,5; \\ Melbourne Genomics Health Alliance, Clara Gaff, PhD ${ }^{2,5}$, Susan M White, MD ${ }^{1,2}$
}

Purpose: To prospectively evaluate the diagnostic and clinical utility of singleton whole-exome sequencing (WES) as a first-tier test in infants with suspected monogenic disease.

Methods: Singleton WES was performed as a first-tier sequencing test in infants recruited from a single pediatric tertiary center. This occurred in parallel with standard investigations, including single- or multigene panel sequencing when clinically indicated. The diagnosis rate, clinical utility, and impact on management of singleton WES were evaluated.

Results: Of 80 enrolled infants, 46 received a molecular genetic diagnosis through singleton WES (57.5\%) compared with $11(13.75 \%)$ who underwent standard investigations in the same patient group. Clinical management changed following exome diagnosis in 15 of 46 diagnosed participants $(32.6 \%)$. Twelve relatives received a genetic diagnosis following cascade testing, and 28 couples were identified as being at high risk of recurrence in future pregnancies.

Conclusions: This prospective study provides strong evidence for increased diagnostic and clinical utility of singleton WES as a firsttier sequencing test for infants with a suspected monogenic disorder. Singleton WES outperformed standard care in terms of diagnosis rate and the benefits of a diagnosis, namely, impact on management of the child and clarification of reproductive risks for the extended family in a timely manner.

Genet Med advance online publication 3 March 2016

Key Words: clinical utility; first tier; monogenic; singleton WES

\section{INTRODUCTION}

Next-generation sequencing (NGS) promises to improve diagnosis and alter patient management by allowing the rapid sequencing of many genes simultaneously at a lower cost compared with sequential testing of multiple genes. ${ }^{1-3}$ Several large studies have demonstrated a diagnostic yield of $25-45 \%$ for clinical whole-exome sequencing (WES). ${ }^{4-7}$ These studies have generally comprised mixed-convenience samples of patients referred for WES, often after substantial prior genetic testing. Recently, the FORGE Canada project reported on the utility of WES after standard-of-care genetic testing in a cohort of Canadian children with unexplained rare diseases. A molecular diagnosis of a known disorder was reached in 105 of 362 families $(29 \%)$, demonstrating the utility of WES used toward the end of the diagnostic process. ${ }^{8}$
These data provide strong evidence that WES should become a critical step in the process of rare genetic disease diagnosis, but there remain unanswered questions regarding its optimal clinical use. First, how does singleton WES perform as a diagnostic test compared with standard care? Second, should WES be applied as a first-tier diagnostic test for all individuals with rare genetic disease or be reserved for complex, undiagnosed individuals, or should it be implemented after metabolic, imaging, and directed genetic testing options have been exhausted?

We sought to answer these questions through a prospective study utilizing singleton WES in parallel with standard diagnostic care in infants with suspected monogenic disorders. Previous studies have investigated cases of unexplained rare diseases or reported on the outcomes of testing a series of unselected patients ascertained through diagnostic laboratories. ${ }^{4-6,8}$ By

The last two authors contributed equally to this work.

${ }^{1}$ Murdoch Childrens Research Institute, Melbourne, Australia; ${ }^{2}$ University of Melbourne, Melbourne, Australia; ${ }^{3}$ Royal Women's Hospital, Melbourne, Australia; ${ }^{4}$ Royal Children's Hospital, Melbourne, Australia; ${ }^{5}$ Melbourne Genomics Health Alliance, Melbourne, Australia; ${ }^{6}$ Royal Melbourne Hospital, Melbourne, Australia. Correspondence: Susan M. White (sue.white@vcgs.org.au)

Submitted 3 November 2015; accepted 23 December 2015; advance online publication 3 March 2016. doi:10.1038/gim.2016.1 
contrast, we investigated patients who had well-defined phenotypes consistent with known genetic conditions to determine the value of applying WES to this frequently encountered set of patients. An infant cohort was chosen because of the diagnostic challenges early in life with clinical presentations that are undifferentiated or incomplete, and because of the opportunity to influence outcomes both for patients and for their relatives of reproductive age. A range of NGS testing strategies have been applied to the molecular diagnosis of pediatric monogenic disorders. ${ }^{9}$ We chose singleton WES rather than NGS panel or trio WES in order to test an approach that balances reduced sequencing costs (in comparison to trios) with the greatest opportunity to expand the genes analyzed at the time of testing based on evolving phenotype information or in response to new gene discoveries.

\section{MATERIALS AND METHODS}

\section{Study design and participants}

Participants were recruited during clinical care by the genetics service at the Royal Children's Hospital ( $\mathrm{RCH})$, Melbourne, Australia, between February 2014 and May 2015, in consultation with their treating clinicians. The patient selection criteria are summarized in Table 1.

We recruited infants ( $0-2$ years of age) who presented with multiple congenital abnormalities and dysmorphic features or other features strongly suggestive of monogenic disorders, for example, neurometabolic conditions and skeletal dysplasias. Infants with specific clinical presentations indicative of conditions that are not genetically heterogeneous (for example, achondroplasia or Apert syndrome) were not considered for the study, except when testing for the suspected disorder was not commercially available. A single-nucleotide polymorphism microarray analysis was a prerequisite for inclusion into the study. Because data analysis was limited to genes currently known to cause monogenic disorders, we excluded infants assessed as having novel phenotypes or orphan conditions.

Potential participants were discussed by a panel of study investigators to determine eligibility. A core data set, including Human Phenotype Ontology terms to record each participant's key features, was collected at enrollment and recorded using PhenoTips. ${ }^{10}$ All diagnostic investigations, including those planned, were collected from referring clinicians and from the medical records at enrollment.

Table 1 Criteria used to select participants for the study

\begin{tabular}{ll} 
Inclusion criteria & \multicolumn{1}{c}{ Exclusion criteria } \\
\hline Infant ( $\leq 2$ years) & $\begin{array}{l}\text { Copy-number variant responsible for } \\
\text { phenotype }\end{array}$ \\
$\begin{array}{l}\text { Multiple congenital abnormalities } \\
\text { and dysmorphic features }\end{array}$ & $\begin{array}{l}\text { Previous single-gene testing } \\
\text { (completed) }\end{array}$ \\
$\begin{array}{l}\text { Other features of monogenic } \\
\text { disorder (e.g., neurometabolic, } \\
\text { renal, eye) }\end{array}$ & $\begin{array}{l}\text { Single-gene disorder unlikely, e.g., } \\
\text { isolated congenital heart disease }\end{array}$ \\
& $\begin{array}{l}\text { Disorder caused by known gene } \\
\text { unlikely, e.g., unique or orphan } \\
\text { phenotype }\end{array}$ \\
\hline
\end{tabular}

A phenotype-driven list of candidate genes for prioritized analysis was generated for each participant by his or her clinician and supplemented by database searches and team discussion at recruitment. Information regarding the impact of the molecular diagnoses reached on patient management was collected from the clinicians involved in each participant's care.

The study was part of the Melbourne Genomics Health Alliance demonstration project (http://www.melbournegenomics.org.au) and received Human Research Ethics Committee approval (13/MH/326). Parents provided written informed consent after genetic counseling regarding the testing. Infants whose families consented to the study underwent WES in parallel with standard investigations, including commercial single- or multigene panel sequencing when clinically indicated and available.

\section{Exome sequencing, variant detection, and variant filtering} DNA was extracted from peripheral blood, and exome sequencing used Nextera v1.2 Rapid Exome Capture Kit and TruSeq Rapid SBS chemistry on a HiSeq 2500 at the Centre for Translational Pathology (University of Melbourne) or the Australian Genome Research Facility. The mean coverage obtained was 145.38 (91.9-203.7). Variants were characterized using the Melbourne Genomics Health Alliance shared bioinformatics pipeline, Cpipe ${ }^{11}$ version 0.9.8 (http://cpipeline.org). Cpipe, a clinically oriented pipeline built on Bpipe, ${ }^{12}$ includes the Burrows-Wheeler Aligner (BWA-0.7.5a-r405) ${ }^{13}$ and the Genome Analysis ToolKit (GATK version 2.8-1-g932cd3a). ${ }^{14}$ Variants were initially filtered against an internal database of variant counts to exclude technical and locally recurrent variants.

Nonsynonymous variants, splice-site variants (up to 10 base pairs), and small insertions and deletions present at a frequency of less than $1 \%$ in the Exome Sequencing Project (http://evs.gs.washington.edu/EVS) and 1000 Genomes Project (http://1000genomes.org) repositories were assessed further. Only variants in HUGO Gene Nomenclature Committee genes associated with Mendelian disease prior to the end of 2013 (2,830 genes) were available for initial analysis. Because WES was not yet an accredited test in Australian laboratories, the analysis pipeline excluded variants in highly penetrant genes predictive of adult-onset diseases such as familial cancer risk genes, cardiovascular disease risk genes, and late-onset neurological disease genes (122 genes). Analysis of one or more of these genes was available only when relevant to the participant's phenotype.

Variants remaining after gene target region filtering were annotated using ANNOVAR (version 2013Aug23), ${ }^{15}$ Condel (version 24-1-2013), ${ }^{16}$ and Variant Effect Predictor (version 74). ${ }^{17}$ Variants were then filtered by variant type, population frequency, and technical frequency. The proportion of gene target regions covered by at least $20 \times$ were summarized in Cpipe's sample quality report. Regions with coverage $<15 \times$ in the target regions were compiled into a gap report by Cpipe.

Variants were assessed using the Melbourne Genomics variant curation database, a modification of the Leiden Open Variation Database. ${ }^{18}$ Variants were prioritized based on the cliniciangenerated gene lists for each participant (Gene Prioritization 
Index) and on predicted effect (Variant Prioritization Index). ${ }^{11}$ Only variants relevant to the participant's phenotype were assessed with regard to pathogenicity. Criteria for classification were based on the principles outlined in the American College of Medical Genetics and Genomics standards for interpretation of sequence variants. ${ }^{19}$ Variant classifications were reviewed in a multidisciplinary team meeting attended by clinical geneticists and other medical subspecialists, genetic counselors, molecular geneticists, and bioinformaticians. Pathogenic and likely pathogenic variants considered to be of clinical significance were confirmed by Sanger sequencing, and, when available, parents were also tested by Sanger sequencing to determine segregation. When additional consent was given, the WES data of unsolved cases were reexamined to identify variants in newly discovered genes matching the participant's phenotype. The processes for participant recruitment and data analysis are summarized in Figure 1.

\section{RESULTS}

\section{Participant demographics and indications for testing}

A total of 119 infants were considered for inclusion, of whom 89 met the selection criteria (Figure 2). Of these, 80 were enrolled in the study, and their characteristics and indications for testing are summarized in Table 2. The average age at enrollment was 8 months (range: 1 week-34 months). Features of the underlying disorder were present at birth in 77 participants (96\%), and in 9 an underlying genetic condition was suspected prenatally. Nine participants died during the study.

The number of genes for prioritized analysis generated by clinicians for each participant at enrollment ranged from 1 to 161 , with a median of 15 .

\section{Diagnostic yield of WES}

WES resulted in a molecular diagnosis of 48 genetic conditions in 46 of 80 infants, for a diagnostic rate of 57.5\% (Supplementary
Table S1 online). Twenty-one of the diagnoses were of a dominant disorder (6 inherited and 15 de novo), 21 were of an autosomal recessive condition, and 3 were of an X-linked condition ( 1 de novo, 2 inherited). The median time to report was 134 days (range: 83-278 days).

\section{Diagnostic yield of standard clinical care}

Twenty-one of 80 participants (26\%) had one or more genetic tests (Sanger sequencing of single genes, NGS gene panels, methylation studies, and mitochondrial mutation panels) as part of standard clinical care during the study period, and a molecular diagnosis was reached for 11 participants (13.75\%) (Supplementary Table S1 online, sections I and IV). All but one of the molecular diagnoses established by standard clinical testing were also identified by WES.

An additional 20 genetic tests were considered at the time of enrollment but were not performed. Reasons clinicians gave for not proceeding with standard testing in parallel with the WES study included funding constraints (particularly the cost of some NGS panels) and the fact that commercially available panels did not contain all the genes of interest for a particular participant. If all proposed tests had been performed, then an additional 11 molecular diagnoses would have been made (Supplementary Table S1 online, section II), allowing for a maximum of 22 participants diagnosable through standard care within the time frame of the study (27.5\%).

\section{Impact on clinical management}

Fifteen of 46 participants diagnosed through WES (32.6\%) had a change in clinical management (Supplementary Table S1 online). Three participants had additional treatment started, one had treatment stopped, and four had modifications to existing treatment regimens. Nine participants had additional surveillance for known complications of their condition, and one

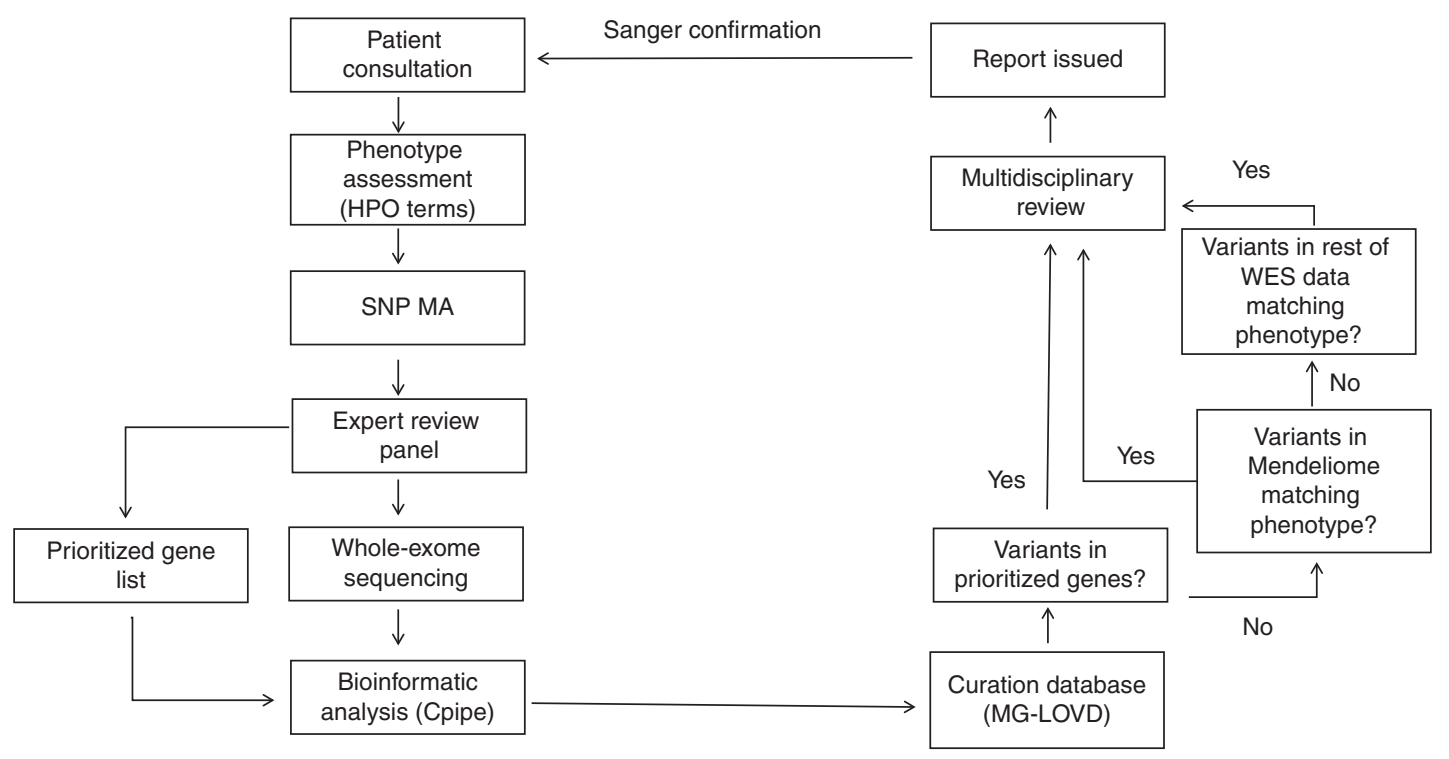

Figure 1 Processes of participant assessment and phenotype-driven WES data analysis used in the study. MA, microarray; MG-LOVD, Melbourne Genomics - Leiden Open Variation Database; SNP, single-nucleotide polymorphism; WES, whole-exome sequencing. 


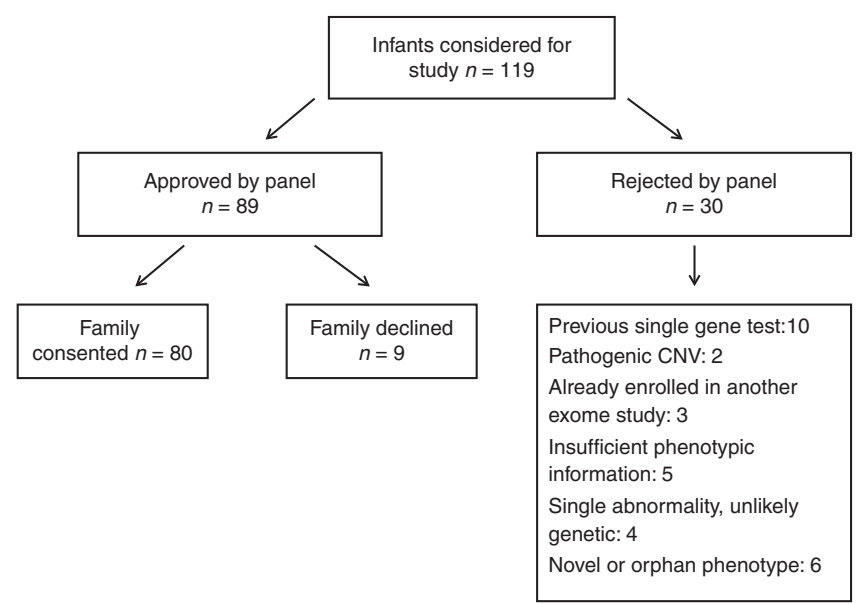

Figure 2 Flowchart of infants recruited into the study. CNV, copynumber variation.

was discharged from surveillance based on an erroneous clinical diagnosis. Eleven of the 15 participants who had a change in clinical management as a result of WES were not diagnosable by standard care in infancy (Supplementary Table S1 online, section III).

Twelve relatives of the infants diagnosed by WES received a genetic diagnosis following cascade testing; only five would have been diagnosed by standard care.

Twenty-eight couples were identified as being at high risk ( 25 or $50 \%)$ of recurrence in future pregnancies as a result of a WES diagnosis for their child. Standard care would have identified 13 of these couples. It identified one additional couple at risk for a recessive condition that was not detected by WES (Supplementary Table S1 online, section IV). WES identified one couple as being at risk for two separate autosomal recessive conditions, but only one of these conditions was identified through standard care.

\section{DISCUSSION}

The application of WES in the clinical setting has the potential to change the paradigm of rare-disease diagnosis. ${ }^{8}$ Most of the large-scale studies of diagnostic and clinical utility of WES have included patients who have undergone substantial prior genetic testing. ${ }^{4-8,20}$ The tendency to use WES late in the diagnostic process may reflect the limited availability of the test, the cost, the backlog of patients with undiagnosed rare diseases, or the desire to maximize opportunities for novel gene discovery. However, the decreasing cost and increasing availability of WES present an opportunity to consider the optimal timing of WES relative to established diagnostic pathways. In the setting of limited resources, it is also important to consider which patient groups are most likely to benefit.

This is the first study to prospectively evaluate the diagnostic and clinical impact of singleton WES as a first-tier sequencing test and compare it with standard care. Singleton WES outperformed standard care in terms of diagnosis rate and the benefits
Table 2 Participant demographics, principal phenotypic features, indications for testing, referral sources, and diagnostic rates of singleton WES in selected groups

\begin{tabular}{|c|c|c|}
\hline Characteristic & $\begin{array}{c}\text { Number } \\
(\%)\end{array}$ & $\begin{array}{l}\text { Diagnostic } \\
\text { rate }\end{array}$ \\
\hline \multicolumn{3}{|l|}{ Sex } \\
\hline Male & $50(62.5 \%)$ & - \\
\hline Female & $30(37.5 \%)$ & - \\
\hline \multicolumn{3}{|l|}{ Age at enrollment } \\
\hline $0-6 \mathrm{~m}$ & $37(46 \%)$ & - \\
\hline $6-12 \mathrm{~m}$ & $25(31 \%)$ & - \\
\hline $12-36 \mathrm{~m}$ & $18(23 \%)$ & - \\
\hline Parental consanguinity & $17(21 \%)$ & $8 / 17(47 \%)$ \\
\hline Affected first-degree relative & $16(20 \%)$ & - \\
\hline Prenatal presentation & $9(11 \%)$ & - \\
\hline \multicolumn{3}{|l|}{ Principal phenotypic feature } \\
\hline $\begin{array}{l}\text { Congenital abnormalities and } \\
\text { dysmorphic features }\end{array}$ & $43(54 \%)$ & $21 / 43(49 \%)$ \\
\hline Neurometabolic disorder & $19(24 \%)$ & $14 / 19(74 \%)$ \\
\hline Skeletal dysplasia & $6(7 \%)$ & $4 / 6(67 \%)$ \\
\hline Eye & $3(4 \%)$ & $2 / 3(67 \%)$ \\
\hline $\begin{array}{l}\text { Other (gastrointestinal, renal, } \\
\text { immunological) }\end{array}$ & $9(11 \%)$ & $5 / 9(55 \%)$ \\
\hline \multicolumn{3}{|l|}{ Primary indication for WES } \\
\hline No clinical diagnosis & $40(50 \%)$ & $16 / 40(40 \%)$ \\
\hline $\begin{array}{l}\text { Clinical diagnosis of a genetically } \\
\text { heterogeneous condition }\end{array}$ & $33(41 \%)$ & $26 / 33(78 \%)$ \\
\hline Suspected dual diagnosis & $3(4 \%)$ & $1 / 3(33 \%)$ \\
\hline Commercial test not available & $4(5 \%)$ & $3 / 4(75 \%)$ \\
\hline \multicolumn{3}{|l|}{ Referral source } \\
\hline Inpatient consultation & $44(55 \%)$ & $24 / 44(54 \%)$ \\
\hline - Neonatal intensive-care unit & $33(41 \%)$ & $16 / 33(48 \%)$ \\
\hline - Pediatric intensive-care unit & $4(5 \%)$ & $3 / 4(75 \%)$ \\
\hline - Other inpatient consultation & $7(8 \%)$ & $5 / 7(71 \%)$ \\
\hline Outpatient consultation & $36(45 \%)$ & $22 / 36(61 \%)$ \\
\hline
\end{tabular}

WES, whole-exome sequencing.

of a diagnosis, namely, impact on management of the child and clarification of reproductive risks for the extended family.

There are concerns that NGS can result in false-negative results due to incomplete coverage and the limited ability to reliably detect variation in repetitive elements and structural variants. In our cohort, singleton WES showed performance comparable to individual-gene Sanger sequencing and diseasespecific NGS panels, with all but one of the molecular diagnoses established through standard care also being established through WES. The overall diagnostic yield of standard care was considerably lower than that of WES (13.75 vs. $57.5 \%)$. It is possible that enrollment into the study-as well as financial constraints-influenced clinician decisions regarding initiating further tests. For this reason, we recorded the additional tests that clinicians considered. Even if all considered tests had proceeded, a diagnosis would have been made in fewer than half of the participants diagnosed through WES (27.5 vs. $57.5 \%)$. The substantial difference in diagnostic rates between traditional 
approaches and WES is similar to that reported in other studies across a broad range of clinical indications..$^{21,22}$

The rate of molecular diagnosis reported here $(57.5 \%)$ demonstrates that singleton WES is a highly successful diagnostic test in infants with monogenic disorders. The higher rate compared with most other reported studies ${ }^{4-8,20}$ can be attributed to the early use of WES in the diagnostic process and the characteristics of the cohort. The large cohorts published by laboratories performing clinical WES include both pediatric and adult patients, ${ }^{5,6}$ with the mean age of the pediatric patients being 6 years. ${ }^{5}$ The primary indication for testing in the pediatric age group was developmental delay, ${ }^{5,6}$ which is a nonspecific phenotype with a very broad differential diagnosis and marked genetic heterogeneity. In addition, patients were not required to undergo standardized clinical examinations or diagnostic testing prior to WES, the phenotypic information provided to laboratories was variable, and prior patient investigations reported by referring physicians were not systematically confirmed. ${ }^{5,6}$ By contrast, the infants included in this study were clinically ascertained, presented with a range of severe phenotypes characterized by structural and other abnormalities, and were phenotyped in detail. The results of previous investigations and the overall likelihood of a monogenic disorder were subject to peer review. These factors are recognized to increase diagnostic yield and/or facilitate data analysis. ${ }^{23}$

Two small studies, of 15 and 35 patients, previously suggested high diagnostic yield in critically ill infants (73 and 55\%, respectively), ${ }^{7,21}$ In the first study, a diagnosis was obtained for $73 \%$ through whole-genome sequencing. ${ }^{7}$ In the subsequent study, a diagnosis was obtained for 55\% through trio wholegenome sequencing. ${ }^{21}$ The use of trio analysis or whole-genome sequencing may increase diagnostic yields compared with singleton WES analysis. ${ }^{6-8}$ However, the fact that we have achieved a very similar diagnostic rate using singleton WES suggests that the common elements of study design between this study and that by Willig et al.- namely, the early timing of the test and the selected study population-are largely responsible for the high diagnostic yield. Within our cohort, a positive correlation between acuity of illness and diagnostic rate was not identified; the diagnostic rate for critically unwell infants (those recruited from intensive-care units) was lower than that for infants recruited following tertiary outpatient assessments (51\% compared with $61 \%$, Table 2 ).

There are several groups of patients in whom the early application of WES was particularly beneficial. The largest group comprised the infants included in the study without a definite clinical diagnosis, mostly reflecting the incomplete and/or undifferentiated nature of their initial presentation. Traditionally, these patients would have been reviewed periodically in the hope that further phenotypic features would emerge over time to enable a diagnosis. This traditional approach may have yielded a diagnosis in a relatively short time in the infant diagnosed with Kabuki syndrome (0204321, Supplementary Table S1 online), who was undiagnosed during the newborn period but had the typical facial features of Kabuki syndrome when reassessed at 18 months. However, it is likely to have taken longer for the infant who presented with microcephaly and developmental delay and was diagnosed with Cohen syndrome (0204435, Supplementary Table S1 online). Cohen syndrome is typically diagnosed in late childhood or adolescence once the features, such as facial gestalt, truncal obesity, neutropenia, and retinal dystrophy, manifest. ${ }^{24}$

The second group of patients in whom early WES was beneficial comprised those who avoided repeated sequential testing. Two infants referred to the study with "no clinical diagnosis" received a diagnosis through WES, which had been included in the differential diagnosis but was not considered the most likely candidate. Another five infants referred with a "clinical diagnosis of a genetically heterogeneous condition" received a diagnosis of a different condition altogether. In the absence of WES, these infants would have undergone testing of the most likely candidate gene(s) first, followed by sequential testing of other candidates over a period of time. This is exemplified by infant 0204350 (Supplementary Table S1 online), who presented with cleft lip and palate, ankyloblepharon, and oral synechiae. The referring clinician suspected ankyloblepharonectodermal defects-cleft lip/palate (AEC) syndrome caused by mutations in TP63. This infant had a previously published de novo pathogenic mutation in IRF6 that caused popliteal pterygium syndrome. ${ }^{25}$ Although this diagnosis was considered part of the differential diagnosis, it was not considered likely in the absence of popliteal pterygia.

There are several reports of blended phenotypes resulting from mutations in more than one gene, ${ }^{4,8}$ and three infants were enrolled with the suspicion of a dual diagnosis. Two of these remain undiagnosed, suggesting that they have a novel phenotype. Two infants received a dual diagnosis during the course of the study. One (0204317) presented with erythema and severe skin desquamation. A diagnosis of Netherton syndrome was initially suspected but was not confirmed on hair biopsies. A skeletal survey identified features of a ciliopathy, and the clinical diagnosis was revised to cranioectodermal dysplasia. WES identified pathogenic mutations in SPINK5 and DYNC2H1, and the dual diagnosis of Netherton syndrome and Jeune asphyxiating thoracic dystrophy was made.

Three infants received a molecular diagnosis upon re-examination of WES data for variants in genes discovered within the time frame of the study (0204307, 0204323, and 0204309; Supplementary Table S1 online, section III). Two of these cases highlight the value of WES for infants with a clinical diagnosis of genetically heterogeneous conditions such as distal arthrogryposis (0204323) and Joubert syndrome (0204309). These infants were found to have mutations in NALCN and KIAA0586, respectively, with both genes having been associated with disease this year. ${ }^{26-29}$ NGS panel containing known disease genes performed for these two infants in 2014 would have returned a negative result. Identifying the causative mutations would have required retesting because new genes were described in the literature and incorporated into commercially available tests. Identifying the causative mutations from existing WES 
data is less resource-intensive and can be performed at shorter time intervals than clinical review of undiagnosed patients. Nevertheless, optimizing the yield of this approach requires the development of robust laboratory and clinical systems for the reexamination of existing data and return of results to families.

Another important group of infants for whom early use of WES is an important avenue to diagnosis are those with lifelimiting disorders. Repeated clinical assessment or multiple rounds of sequential testing may not be possible for these infants. Six of nine $(67 \%)$ deceased infants received a WES diagnosis (versus two using standard testing).

The value of WES in enabling the diagnosis of ultra-rare conditions, which are unlikely to be considered clinically, has recently been highlighted. ${ }^{8}$ One such diagnosis was made in our cohort: immunodeficiency type $31 \mathrm{~B}$ caused by a homozygous loss-offunction STAT1 mutation (infant 0204409). This condition has been reported only three times in the literature $\mathrm{e}^{30-32}$ and was not considered a differential diagnosis by the referring clinicians.

The high diagnostic yield obtained in this cohort has had marked clinical utility for participants, families, and treating medical teams. For all infants in whom a diagnosis was made, accurate prognostic information could be provided to their families and medical teams. For seven infants, the molecular diagnosis obtained through WES was substantially different from the provisional clinical diagnosis initially discussed with the family, resulting in new, more accurate information being provided. All infants receiving a molecular diagnosis through WES avoided further diagnostic investigations; this included invasive tissue biopsies that were planned for three infants with suspected mitochondrial disease (0204307, 0204313, 0204320).

One-third of diagnosed infants had a change in clinical management as a result of WES diagnosis. Three had additional treatment started, including infant 0204320, who presented with developmental delay, hypotonia, and seizures and had a lactate peak on magnetic resonance spectroscopy. A clinical diagnosis of Leigh disease was made, and the parents were counseled that this was likely to be life-limiting. He was found to have compound heterozygous mutations in SLC19A3. This gene encodes a thiamine transporter, and mutations cause an infantile Leigh-like phenotype. ${ }^{33}$ Thiamine supplementation was commenced and so far he has not experienced further deterioration. ${ }^{34}$ Five participants had treatments stopped or modified, including infant 0204339 , who had a clinical diagnosis of osteogenesis imperfecta type $\mathrm{V}$ and received bisphosphonate infusions. He was found to have a previously reported de novo heterozygous pathogenic mutation in $\mathrm{NOTCH} 2$ that causes Hajdu-Cheney syndrome. ${ }^{35}$ The number of participants with altered clinical management after WES $(N=15,32.6 \%)$ is lower than that reported by Willig et al., ${ }^{21}$ who found that rapid whole-genome sequencing for acutely unwell infants was clinically useful for $65 \%$ of diagnosed patients, but higher than that reported by Sawyer et al., ${ }^{8}$ who described a change in management in 6 of 105 (5.7\%) patients diagnosed near the end of the diagnostic odyssey. It is likely that these differences are related to the timing of the test (start versus end of diagnostic journey) and the speed with which the result is available. The median time to report in our study was 134 days (83-278), compared with the median time to report of 23 days (5-912) in the study by Willig et al. ${ }^{21}$

The benefits of molecular diagnosis have extended beyond the direct management of the study participants. Cascade testing in first-degree relatives resulted in a molecular diagnosis being made in five siblings and seven parents. Two of the parents and one of the siblings were not clinically suspected of being affected prior to the diagnosis being made for the proband. This led to further management initiated in these family members on the basis of the exome result.

A molecular diagnosis in the participants enabled the provision of accurate recurrence advice to their parents, who are all of reproductive age. The 28 families advised of a high recurrence risk (either 25 or $50 \%$ ) in future pregnancies now have the option of prenatal or preimplantation genetic diagnosis available if they wish to reduce their risk. Within the short period of the study, five of the families who received a diagnosis have embarked on a new pregnancy, with three utilizing prenatal diagnosis.

This study was limited by the absence of a randomized masked control group, which would have allowed the comparison of further utility parameters, such as time to diagnosis, and removed possible clinician bias toward not initiating new genetic investigations while WES was in progress. The deliberate exclusion of infants with presumed novel phenotypes means that these findings cannot be extrapolated across all infants with suspected monogenic disorders, and it is clear that this challenging patient group require further research to determine the optimal NGS approach to diagnosis in them. Assessment of the usefulness of WES as a first-line sequencing test would benefit from measurements of other outcomes, including costeffectiveness, health and psychosocial impacts for participating families, and reproductive outcomes, as well as evaluation of other patient groups.

Our study provides evidence that using singleton WES as a first-line sequencing test for infants can considerably shorten and simplify the diagnostic process by enabling diagnostic testing to occur at a much earlier point and eliminating sequential testing of candidate genes. WES provides a higher diagnostic yield than standard testing by facilitating the diagnosis of conditions that are considered unlikely or not considered at all by clinicians, those subject to extreme genetic heterogeneity, and conditions caused by recently discovered genes, as well as by resolving phenotypes due to more than one genetic condition. The adoption of WES as a first-line sequencing test in infants presenting with features suggestive of a monogenic disorder results in a high proportion of patients receiving an accurate diagnosis early in the clinical course, which maximizes opportunities to improve clinical outcomes for patients and families.

\section{SUPPLEMENTARY MATERIAL}

Supplementary material is linked to the online version of the paper at http://www.nature.com/gim 


\section{ACKNOWLEDGMENTS}

This study was funded by the members of the Melbourne Genomics Health Alliance and the State Government of Victoria (Department of Health and Human Services). We thank the patients and families for participating in this study. We are grateful to Rodney Hunt, Amanda Moody, David Tingay, John Mills, Michael Stewart, Anastasia Pellicano, Leah Hickey, Julia Gunn, Margaret Moran, Mark Mackay, Avihu Boneh, and Cathy Quinlan for referring patients to the study; Kate Pope for genetic counseling support; Nessie Mupfeki for data-management support; and Harriet Dashnow, Gayle Phillip, Anthony Marty, and Andrew Lonie for bioinformatics and database-development support. We also acknowledge the Melbourne Genomics Health Alliance Steering Group, the Clinical Genomics and Bioinformatics Advisory Group, and the Clinical Genomics Advisory Group for establishing the systems and standards applied in this study.

\section{DISCLOSURE}

The authors declare no conflict of interest.

\section{REFERENCES}

1. Boycott KM, Vanstone MR, Bulman DE, MacKenzie AE. Rare-disease genetics in the era of next-generation sequencing: discovery to translation. Nat Rev Genet 2013;14:681-691.

2. Dixon-Salazar TJ, Silhavy JL, Udpa N, et al. Exome sequencing can improve diagnosis and alter patient management. Sci Trans/ Med 2012;4:138ra78.

3. Saunders CJ, Miller NA, Soden SE, et al. Rapid whole-genome sequencing for genetic disease diagnosis in neonatal intensive care units. Sci Trans/ Med 2012;4:154ra135

4. Yang Y, Muzny DM, Reid JG, et al. Clinical whole-exome sequencing for the diagnosis of Mendelian disorders. N Engl J Med 2013;369:1502-1511.

5. Yang Y, Muzny DM, Xia F, et al. Molecular findings among patients referred for clinical whole-exome sequencing. JAMA 2014;312:1870-1879.

6. Lee $\mathrm{H}$, Deignan JL, Dorrani N, et al. Clinical exome sequencing for genetic identification of rare Mendelian disorders. JAMA 2014;312:1880-1887.

7. Soden SE, Saunders CJ, Willig LK, et al. Effectiveness of exome and genome sequencing guided by acuity of illness for diagnosis of neurodevelopmental disorders. Sci Transl Med 2014;6:265ra168.

8. Sawyer SL, Hartley T, Dyment DA, et al.; FORGE Canada Consortium; Care4Rare Canada Consortium. Utility of whole-exome sequencing for those near the end of the diagnostic odyssey: time to address gaps in care. Clin Genet 2016;89:275-84.

9. Beaulieu CL, Majewski J, Schwartzentruber J, et al.; FORGE Canada Consortium. FORGE Canada Consortium: outcomes of a 2-year national rare-disease genediscovery project. Am J Hum Genet 2014;94:809-817.

10. Girdea M, Dumitriu S, Fiume M, et al. PhenoTips: patient phenotyping software for clinical and research use. Hum Mutat 2013;34:1057-1065.

11. Sadedin SP, Dashnow H, James PA, et al.; Melbourne Genomics Health Alliance. Cpipe: a shared variant detection pipeline designed for diagnostic settings. Genome Med 2015;7:68.

12. Sadedin SP, Pope B, Oshlack A. Bpipe: a tool for running and managing bioinformatics pipelines. Bioinformatics 2012;28:1525-1526.

13. Li H, Durbin R. Fast and accurate long-read alignment with Burrows-Wheeler transform. Bioinformatics 2010;26:589-595.

14. Van der Auwera GA, Carneiro MO, Hartl C, et al. From FastQ data to high confidence variant calls: the Genome Analysis Toolkit best practices pipeline. Curr Protoc Bioinformatics 2013;11:11.10.1-11.10.33.
15. Wang K, Li M, Hakonarson H. ANNOVAR: functional annotation of genetic variants from high-throughput sequencing data. Nucleic Acids Res 2010;38:e164.

16. González-Pérez A, López-Bigas N. Improving the assessment of the outcome of nonsynonymous SNVs with a consensus deleteriousness score, Condel. Am J Hum Genet 2011;88:440-449.

17. McLaren W, Pritchard B, Rios D, Chen Y, Flicek P, Cunningham F. Deriving the consequences of genomic variants with the Ensembl API and SNP Effect Predictor. Bioinformatics 2010;26:2069-2070.

18. Fokkema IF, Taschner PE, Schaafsma GC, Celli J, Laros JF, den Dunnen JT. LOVD v.2.0: the next generation in gene variant databases. Hum Mutat 2011;32: 557-563.

19. Richards CS, Bale S, Bellissimo DB, et al.; Molecular Subcommittee of the ACMG Laboratory Quality Assurance Committee. ACMG recommendations for standards for interpretation and reporting of sequence variations: revisions 2007. Genet Med 2008;10:294-300.

20. Wright CF, Fitzgerald TW, Jones WD, et al.; DDD study. Genetic diagnosis of developmental disorders in the DDD study: a scalable analysis of genome-wide research data. Lancet 2015;385:1305-1314.

21. Willig LK, Petrikin JE, Smith LD, et al. Whole-genome sequencing for identification of Mendelian disorders in critically ill infants: a retrospective analysis of diagnostic and clinical findings. Lancet Respir Med 2015;3:377-387.

22. Neveling K, Feenstra I, Gilissen C, et al. A post-hoc comparison of the utility of sanger sequencing and exome sequencing for the diagnosis of heterogeneous diseases. Hum Mutat 2013;34:1721-1726.

23. Boycott K, Hartley T, Adam S, et al.; Canadian College of Medical Geneticists. The clinical application of genome-wide sequencing for monogenic diseases in Canada: position statement of the Canadian College of Medical Geneticists. J Med Genet 2015;52:431-437.

24. El Chehadeh-Djebbar S, Blair E, Holder-Espinasse M, et al. Changing facial phenotype in Cohen syndrome: towards clues for an earlier diagnosis. Eur J Hum Genet 2013;21:736-742.

25. Kondo S, Schutte BC, Richardson RJ, et al. Mutations in IRF6 cause Van der Woude and popliteal pterygium syndromes. Nat Genet 2002;32:285-289.

26. Alby $C$, Piquand K, Huber $C$, et al. Mutations in KIAA0586 cause lethal ciliopathies ranging from a hydrolethalus phenotype to short-rib polydactyly syndrome. Am J Hum Genet 2015;97:311-318.

27. Bachmann-Gagescu R, Phelps IG, Dempsey JC, et al.; University of Washington Center for Mendelian Genomics. KIAA0586 is mutated in Joubert syndrome. Hum Mutat 2015;36:831-835.

28. Chong JX, McMillin MJ, Shively KM, et al.; University of Washington Center for Mendelian Genomics. De novo mutations in NALCN cause a syndrome characterized by congenital contractures of the limbs and face, hypotonia, and developmental delay. Am J Hum Genet 2015:96:462-473.

29. Roosing S, Hofree M, Kim S, et al. Functional genome-wide siRNA screen identifies KIAA0586 as mutated in Joubert syndrome. Elife 2015;4:e06602.

30. Chapgier A, Wynn RF, Jouanguy E, et al. Human complete Stat-1 deficiency is associated with defective type I and II IFN responses in vitro but immunity to some low virulence viruses in vivo. J Immunol 2006;176:5078-5083.

31. Dupuis S, Jouanguy E, Al-Hajjar S, et al. Impaired response to interferonalpha/beta and lethal viral disease in human STAT1 deficiency. Nat Genet 2003;33:388-391.

32. Kong XF, Ciancanelli M, Al-Hajjar S, et al. A novel form of human STAT1 deficiency impairing early but not late responses to interferons. Blood 2010;116:5895-5906.

33. Kevelam SH, Bugiani M, Salomons GS, et al. Exome sequencing reveals mutated SLC19A3 in patients with an early-infantile, lethal encephalopathy. Brain 2013;136:1534-1543.

34. Haack TB, Klee D, Strom TM, et al. Infantile Leigh-like syndrome caused by SLC19A3 mutations is a treatable disease. Brain 2014;137:e295.

35. Simpson MA, Irving MD, Asilmaz E, et al. Mutations in NOTCH2 cause HajduCheney syndrome, a disorder of severe and progressive bone loss. Nat Genet 2011;43:303-305. 